Check for updates

Cite this: Mater. Adv., 2021, 2, 4352

Received 22nd February 2021, Accepted 12th May 2021

DOI: $10.1039 / \mathrm{d} 1 \mathrm{ma} 00155 \mathrm{~h}$

rsc.li/materials-advances

\title{
A synergistic approach to achieving the high thermoelectric performance of La-doped SnTe using resonance state and partial band convergence $\dagger$
}

\author{
Srikanth Mandava, ${ }^{a}$ Ranita Basu, (D) *b B. Khasimsaheb, ${ }^{c}$ Sivaiah Bathula, de \\ Sai Muthukumar V., Ajay Singh ${ }^{b}$ and S. Neeleshwar ${ }^{a}$
}

\begin{abstract}
$\mathrm{SnTe}$ is an alternate variant of PbTe possessing an analogous valence band (VB) pattern. However, $\mathrm{SnTe}$ exhibits low thermoelectric (TE) efficiency due to $\mathrm{Sn}$ defects triggering very high carrier concentration (n). Thus, $\mathrm{Sn}_{1-x} \mathrm{La}_{x} \mathrm{Te},(x=0.00,0.04,0.06,0.08)$ was doped with lanthanum (La) to control the vacancy related defects. The TE performance of SnTe was considerably enhanced, where La acts as an electron donor. We propose that La at Sn sites in SnTe creates resonance levels at the VB due to hybridization of the $6 s$ orbital of La and the $5 p$ orbital of Te atom to form the $s-p$ antibonding state, thereby enhancing the Seebeck coefficient and power factor. Besides, due to all-length scale hierarchical architecture, $\mathrm{Sn}_{0.94} \mathrm{La}_{0.06} \mathrm{Te}$ gives $\sim 96 \%$ higher calculated figure-of-merit $(z T)$ than pristine SnTe. The device $z T$ value of $\mathrm{Sn}_{0.92} \mathrm{La}_{0.08} \mathrm{Te}$ is $\sim 88 \%$ higher than that of SnTe. Thus, the significant improvement in device efficiency is encouraging for considering these materials as a suitable alternative to $\mathrm{Pb}$-based chalcogenides.
\end{abstract}

\section{Introduction}

The ever increasing demand for energy has led to the search for alternate sources of renewable resources. Globally, approximately $65 \%$ of the total energy of various resources is rejected as waste heat to the environment and that causes global warming. This considerable amount of waste heat can be converted into clean energy by using thermoelectric devices. ${ }^{1}$ The conversion efficiency of thermoelectric materials merely depends on their dimensionless figure-of-merit $(z T)$ of the materials which is given by eqn (1):

$$
z T=\left[S^{2} \sigma /\left(\kappa_{\mathrm{e}}+\kappa_{\mathrm{L}}\right)\right] T
$$

where $T$ is the absolute temperature, $\kappa_{\mathrm{L}}$ is the lattice thermal conductivity, $\kappa_{\mathrm{e}}$ is the electronic thermal conductivity, $\sigma$ is the

${ }^{a}$ Guru Gobind Singh Indraprastha University, Delhi-110078, India

${ }^{b}$ Technical Physics Division, Bhabha Atomic Research Centre, Mumbai-400085, India.E-mail: ranitapaul@gmail.com, ranitab@barc.gov.in

${ }^{c}$ Institute of Physics, Academia Sinica, Taipei 11529, Taiwan

${ }^{d}$ Division of Advanced Materials and Devices Metrology, CSIR-National Physical Laboratory, Dr K. S. Krishnan Marg, New Delhi 110012, India

${ }^{e}$ School of Minerals, Metallurgical and Materials Engineering,

IIT Bhubaneswar-752050, Odisha, India

${ }^{f}$ Sri Sathya Sai Institute of Higher Learning, Vidyagiri Prasanthi Nilayam, Andhra Pradesh 515134, India

$\dagger$ Electronic supplementary information (ESI) available. See DOI: 10.1039/d1ma00155h electrical conductivity, and $S$ is the Seebeck coefficient. In general, an efficient thermoelectric material should have a high Seebeck coefficient, high electrical conductivity and low thermal conductivity. However, these parameters are interlinked and de-coupling of these parameters is the only option to achieve high thermoelectric performance. For most of the state-of-the thermoelectric materials, the $z T$ was limited to $\sim 1$ for many years due to internally connected parameters such as carrier concentration $(n)$, mobility $(\mu)$, and effective mass $\left(m^{*}\right)$, etc. ${ }^{1-4}$ Nevertheless, thermal and electrical conductivities are directly related in the case of metals and these properties can be altered for semiconductors by suitable doping. ${ }^{1-8}$ Also, the Seebeck coefficient and electrical conductivity vary reciprocally, causing difficulties in achieving any drastic improvement in the $z T$. Hence, most of the state-of-the-art thermoelectric materials fall in the category of semiconductors, where their electronic and thermal transport properties can be easily tunable in comparison to metallic systems. ${ }^{7,8}$ Furthermore, enhancement in material performance has to be complemented with improvement in the module design to achieve a higher device level $z T$.

A large number of narrow bandgap materials are aimed at room temperature $(300 \mathrm{~K})$ to high temperatures $(1100 \mathrm{~K})$ for thermoelectric applications. Among them, in the mid-temperature (600-900 K) range, lead chalcogenides (PbTe, PbSe, and PbS) and their solid solutions are the most efficient thermoelectric materials, with the maximum $z T$ of above $2 .{ }^{9,10}$ Despite their 
excellent thermoelectric properties, the perceived toxicity of lead chalcogenides discourage their development and largescale application. In this context, SnTe, which resembles PbTe in many respects such as crystal structure, ${ }^{11}$ narrow bandgap, two valence band characters ${ }^{12-14}$ has attracted researchers worldwide during the last decade. Also, regarding the structural properties, there is much similarity between the SnTe and PbTe, such as microhardness $0.78 \mathrm{GPa}$ and $0.76 \mathrm{GPa},{ }^{15,16}$ Young's moduli $55.6 \mathrm{GPa}$ and $58.1 \mathrm{GPa},{ }^{15,16}$ and Shear moduli $21.7 \mathrm{GPa}$ and $23.0 \mathrm{GPa}^{15,16}$ for SnTe and PbTe, respectively. However, SnTe exhibits a low Seebeck coefficient and high electrical and thermal conductivities owing to high hole concentration which inhibits the pristine material to become an outstanding thermoelectric material. But, there are strategies such as band convergence or resonance effect which lead to the significant increase of $z T$ by power factor enhancement using suitable dopants.

SnTe is a narrow bandgap (p-type) $(\sim 0.18 \mathrm{eV})$ that belongs to the IV-VI family semiconductor. ${ }^{17}$ Analogous to PbTe, SnTe exhibits two valence bands (VB) (L and $\Sigma$ ). However, the large energy separation $(\Delta E=0.35 \mathrm{eV})^{18-20}$ between the light and heavy holes inhibit the $\Sigma$ band to contribute to the transport properties at room temperature whereas, at high temperature, the $\mathrm{L}$ band moves toward the $\Sigma$ band, and thus band convergence occurs. ${ }^{18-23}$ Pei et al. have explained explicitly that in the two-valence band model of PbTe, the energy offset between conduction band $(\mathrm{CB})$ and L-band of $\mathrm{VB}$ is temperaturedependent. However, the energy offset between $\mathrm{CB}$ and the heavy $\Sigma$ band of VB is independent of temperature but moves gradually with the addition of the dopants. ${ }^{24}$ Guo et al. elucidated that the $\mathrm{L}$ valence band of SnTe is dominated by the antibonding state of Sn-s and Te-p orbitals. However, any addition of dopants weakens the strength of the antibonding state of pristine SnTe leading to the decrease in the energy offset between L- and $\Sigma$-valence bands. Thus, doping may induce a band convergence effect in SnTe. ${ }^{25}$ Fortunately, band convergence is beneficial as it increases the density of state effective mass $\left(m_{\mathrm{b}}^{*}\right)$ of bands near the Fermi level $E_{\mathrm{F}}$ and improves the Seebeck coefficient significantly. ${ }^{26,27}$ On the other hand, yet another effect known as the resonance effect may be induced at room temperature when doped with certain elements as the impurity states are created by dopants near the $E_{\mathrm{F}}$ which fall either above the conduction band (CB) edge or below the valence band (VB) edge. ${ }^{14}$ As this state has the same energy as an extended state, the two states resonate to build up to two extended states of slightly different energies; these, in turn, will have the same energies as other extended states with whom they will resonate in turn, 28 and so on as shown in Fig. 1. Thus, the resonant state develops a certain width $\Gamma{ }^{28}$ Stoichiometric SnTe is a p-type semiconductor with a very high carrier concentration resulting from an extremely large number of Sn lattice vacancies, leading to a low Seebeck coefficient. ${ }^{29}$ Moreover, large energy separation between its light and heavy hole bands, $\Delta E=0.3-0.4 \mathrm{eV}$ at room temperature, significantly suppresses the contribution of heavy holes to the Seebeck coefficient at high temperatures. Zhou et al. calculated the light and heavy valence band properties as a function of chemical potential using full generalized Fermi integrals. ${ }^{30}$ The light band was assumed to be a nonparabolic, Kane band, with a non-parabolicity parameter, $\beta$, given by, $\frac{\kappa_{\beta} T}{E_{\mathrm{g}}}$, where $E_{\mathrm{g}}$ was assumed to be constant at $0.18 \mathrm{eV}$, while the heavy band was modeled as a parabolic band. However, transport properties can be tailored by optimizing the carrier concentration using suitable dopants. Thus, numerous attempts have been made to modify the valence band structure of SnTe resulting in a high $z T$ value. ${ }^{31}$ Zhang et al. registered a resonant frequency below the SnTe Fermi level that was caused by indium doping and observed a significant rise in the Seebeck coefficient. ${ }^{32}$ However, Tan et al. indicated that doping should be optimized because with the increase in the doping level, the overall s-p antibonding state is enhanced and thus the resonant level is shifted upward. Consequently, the band convergence effect is hindered.33 Although the concept of valence band convergence in $\mathrm{PbTe}$ with increasing temperature and modification of the band structure in $\mathrm{PbTe}$ with a sharp peak in the density-of-states (DOS) using La as the dopant, which might be considered as a resonant state, was recognized earlier, ${ }^{34}$ using the same analogy, the current study involves doping of SnTe using $\mathrm{La}^{35}$ which considerably enhances the thermoelectric performance using two successive approaches.

To the best of the knowledge, there is no report available on the solid solution synthesis of $\mathrm{Sn}_{1-x} \mathrm{La}_{x} \mathrm{Te}$ for enhancing the thermoelectric performance. Here we have investigated the $\mathrm{Sn}_{1-x} \mathrm{La}_{x} \mathrm{Te}(x=0,0.04,0.06$, and 0.08$)$ compound by a vacuum melting method followed by the spark plasma sintering (SPS). Employing the current methodology, the device $z T \sim 0.17$ at $823 \mathrm{~K}$ has been observed with increasing La content in $\mathrm{Sn}_{0.92} \mathrm{La}_{0.08} \mathrm{Te}$, which is about $88 \%$ higher than the value 0.09 at $775 \mathrm{~K}$ of pristine SnTe. The device $z T$ calculated for $\mathrm{Sn}_{0.94} \mathrm{La}_{0.06} \mathrm{Te}$ and $\mathrm{Sn}_{0.92} \mathrm{La}_{0.08} \mathrm{Te}$ are close to each other.

\section{Experimental procedure}

\subsection{Starting materials}

Chemicals were used as obtained: Sn chunk (99.9999\%, Sigma Aldrich), Te shot (99.999\%, 5 N, Sigma Aldrich), and lanthanum metal $(99.999 \%, 5 \mathrm{~N}$, Alfa aesar).

\subsection{Synthesis}

High-purity single elements $\mathrm{Sn}, \mathrm{Te}$, and La were weighed according to the stoichiometric compositions of $\mathrm{Sn}_{1-x} \mathrm{La}_{x} \mathrm{Te}(x=0,0.04,0.06$, and 0.08 ) and then put inside $13 \mathrm{~mm}$ diameter fused quartz tubes. The tubes were sealed under a vacuum $\left(\sim 10^{-4}\right.$ torr $)$ and slowly heated to $1223 \mathrm{~K}$ at $50 \mathrm{~K}$ per hour followed by rocking for 5 hours to form a uniform composition and then soaked for $10 \mathrm{~h}$ for complete homogenization. The ampoules were then slowly cooled to room temperature at a rate of $70 \mathrm{~K}$ per hour. The obtained ingots were then crushed and ground into powders inside the glove box $(<1 \mathrm{ppm}$ oxygen level) using a mortar pestle for compaction. The ground powders were filled into a $12 \mathrm{~mm}$ diameter graphite die and sintered by spark plasma sintering (SPS, M/s SPS Syntex, 725, Japan) at $773 \mathrm{~K}$ for $\mathrm{Sn}_{1-x} \mathrm{La}_{x} \mathrm{Te}(x=0$ 


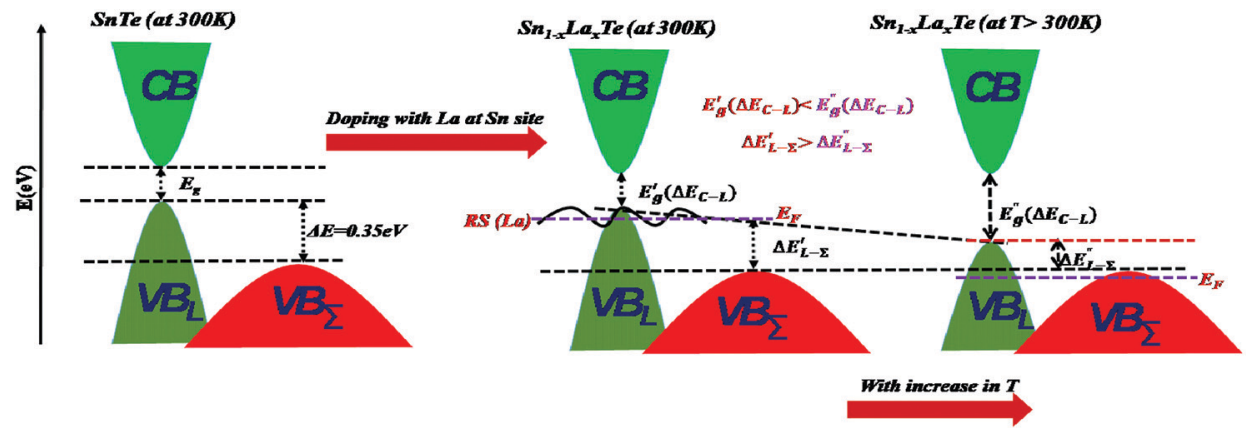

Fig. 1 Schematic band structure of SnTe and La doped SnTe at $300 \mathrm{~K}$ and at high temperature.

and 0.04$)$ and $823 \mathrm{~K}$ for $\mathrm{Sn}_{1-x} \mathrm{La}_{x} \mathrm{Te}(x=0.06$ and 0.08$)$ by maintaining a soaking time of 5 minutes under $40 \mathrm{MPa}$ load with a vacuum $10 \mathrm{~Pa}$. Also, during SPS, the DC pulse ratio (current on/off time in milliseconds) 12:2 has been maintained with a high heating rate of $200{ }^{\circ} \mathrm{C} \mathrm{min}^{-1}$.

\subsection{X-ray diffraction and electron microscopy}

The before and after sintered pellets were characterized by X-ray diffraction (XRD) technique Proto AXRD Benchtop Powder diffractometer), under ambient conditions using $\mathrm{CuK}_{\alpha}$ radiation $(\lambda=1.5418 \AA$ ). Furthermore, a scanning electron microscope (SEM, M/s JEOL IT300 operated at $15 \mathrm{kV}$ ) is used to study the morphological studies and the attached EDS analyzer (M/s EDAX-Octane) is used for elemental analysis and mapping. The transmission electron microscope (JEOL JEM2100F) specimens were created by dispersing the specimen powder onto a copper grid.

\subsection{Electrical transport properties}

The obtained spark plasma sintered (SPS)-processed pellets were cut into bars with dimensions of $12 \mathrm{~mm} \times 3 \mathrm{~mm} \times 3 \mathrm{~mm}$ that was used for simultaneous measurement of the Seebeck coefficient and the electrical conductivity using M/s Linseis LSR-3 instrument under a helium atmosphere from room temperature to $823 \mathrm{~K}$. No hysteresis was seen in heating and cooling cycles. Electrical properties measured for different slices cut from the same pellets were similar, corroborating the homogeneity of the samples. The uncertainty of the Seebeck coefficient and electrical conductivity measurements is $\pm 3 \%$.

\subsection{Thermal conductivity}

Highly dense SPS-processed pellets of diameter $12 \mathrm{~mm}$ were polished for thermal diffusivity measurements. The samples were coated with a thin layer of graphite to minimize errors from the emissivity of the material. The thermal conductivity was calculated from $\kappa=D \times C_{\mathrm{p}} \times \rho$, where the thermal diffusivity coefficient $(D)$ was measured using the laser flash diffusivity method in a Linseis, LFA-1000, the specific heat capacity $\left(C_{\mathrm{p}}\right)$ was determined by eqn (2), ${ }^{30}$

$$
C_{\mathrm{P}}=C_{\mathrm{P}, 300+} C_{\mathrm{P}, 1} \times \frac{\left(\left(\frac{T}{300}\right)^{\alpha}-1\right)}{\left(\frac{T}{300}\right)}+\frac{C_{\mathrm{P} 1}}{C_{\mathrm{p}, 300}}
$$

where $T$ is the absolute temperature and $C_{\mathrm{P}, 300}$ is the specific heat capacity at $300 \mathrm{~K}$. For SnTe, $C_{\mathrm{P}, 300}$ is $0.1973 \mathrm{~J} \mathrm{~g}^{-1} \mathrm{~K}^{-1}, C_{\mathrm{P} 1}$ is $0.115 \mathrm{~J} \mathrm{~g}^{-1} \mathrm{~K}^{-1}$, and $\alpha$ is 0.63 and the density $(\rho)$ was determined using the dimensions and mass of the sample and found to be $>98 \%$ of its theoretical density. ${ }^{29}$ The thermal diffusivity data were analyzed using a Cowan model with pulse correction. The uncertainties in transport and other measurements are $\pm 6 \%$ for $D$, $\pm 3 \%$ for $\sigma, \pm 3 \%$ for $S, \pm 10 \%$ for $C_{\mathrm{p}}$ and $\pm 0.5 \%$ for $\rho$. Unless otherwise mentioned, all the properties described in this study were measured perpendicular to the sintering pressure direction, although no directional anisotropy effects were perceived in the charge transport properties.

\section{Results and discussion}

\subsection{Structural characterization}

As synthesized $\mathrm{Sn}_{1-x} \mathrm{La}_{x} \mathrm{Te}(x=0,0.04,0.06$, and 0.08$)$ were initially characterized by employing XRD to confirm the crystallite size and phase purity. The presence of multiple peaks in the XRD plot suggests the sample is polycrystalline. The Fig. 2(a) of $\mathrm{Sn}_{1-x} \mathrm{La}_{x} \mathrm{Te}(x=0,0.04,0.06$, and 0.08$)$ samples were in good agreement with the standard data (JCPDF 08-0487) confirming the phase purity. It is to be noted that all the Bragg's reflections of samples correspond to SnTe which indicates the occupancy of La in the Sn-site in the lattice. No secondary phases were detected in XRD. Besides, all the samples represent the cubic structure with a space group: $F m \overline{3} m$. Lattice parameters were calculated for the samples and it was found that due to the occupancy of $\mathrm{La}^{3+}$ into $\mathrm{Sn}^{2+}$, the stress so developed causes an increase in the lattice parameter. From Fig. 2(b), it has been
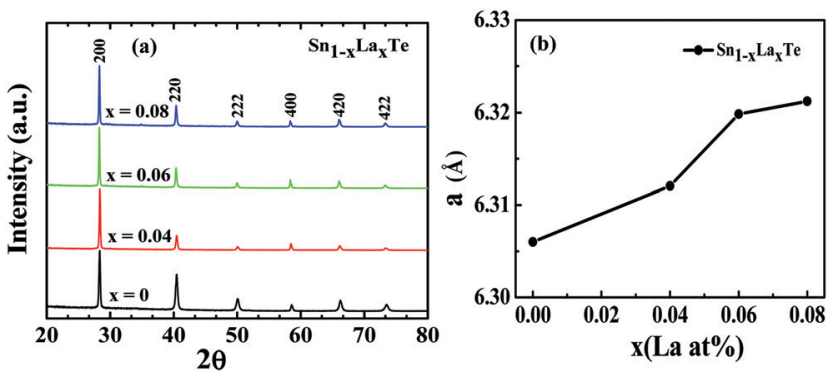

Fig. 2 (a) X-Ray diffraction patterns of $\mathrm{Sn}_{1-x} \mathrm{La}_{x} T e(x=0,0.04,0.06$, and 0.08 ) samples (b) the lattice parameter of different La contents in SnTe. 
observed that when concentration was plotted against the lattice parameter obtained from the XRD plot, there is a volume expansion in the lattice with increasing La concentration. However, the change in the lattice parameter shows a negligible change (deviation from Vegard's law type behavior) as the content reaches 8 at\%. Thus, the increase in the lattice parameter is expected up to the solubility limit of La in SnTe and a similar kind of behavior is observed earlier for other state-ofthe-art thermoelectric materials. ${ }^{10}$ Generally, if $\mathrm{La}_{3} \mathrm{Te}_{4}$ were forming solid solutions with SnTe, one would expect a perpetual Vegard's law type behavior, showing a systematic increase in the band gap with the $\mathrm{La}_{3} \mathrm{Te}_{4}$ amount $\left(E_{\mathrm{g}, \mathrm{La}_{3} \mathrm{Te}_{4}} \sim 0.95 \mathrm{eV}\right)$. The increase in the band-gap is observed only up to 6 at $\%$ fractions. It suggests that for $\mathrm{La},>6$ at $\%$ it is excluded from the SnTe matrix. Collectively, both the lattice parameter and bandgap variations suggest that the solubility limit is somewhere around 6 at\%.

The SEM images revealing the surface morphology of (a) SnTe and (d) $\mathrm{Sn}_{0.92} \mathrm{La}_{0.08} \mathrm{Te}$ samples after SPS are shown in Fig. 3. The image shows that the samples exhibit dense microstructure. The SEM image reveals that kink bands are clearly visible. Moreover, these kink bands have kink planes which are seen up to few microns and are nearly parallel to each other. The uniqueness of the planes is that they are the most active planes for the slip to occur and arises due to the anisotropic growth of the sample. ${ }^{21}$ The study by Yang et $a l .{ }^{36}$ reported that these kink bands serve as scattering centres for the phonons and thus suppress the thermal conductivity (Fig. 3(d)). Interestingly, a similar kind of morphology is exhibited by all the samples even with increasing La doping in SnTe. However, a nanoprecipitate is seen in the microstructure when the concentration of $x=0.08$. This can be attributed to the limited solubility of La in SnTe. This nanoprecipitate is absent in the XRD plot which can be due to the low concentration which is beyond the detection limit. EDS mapping of samples confirm the single-phase compound for $\mathrm{Sn}_{1-x} \mathrm{La}_{x} \mathrm{Te}(x=0,0.04$ and 0.06). However, from Fig. 3(g), it has been observed that there is a sign of $\mathrm{La}$ precipitation in the case of doping when $\mathrm{La}$ is present in 8 at\%. The EDS spectra of all the compositions of La-doped SnTe, $\mathrm{Sn}_{1-x} \mathrm{La}_{x} \mathrm{Te}(x=0,0.04,0.06$ and 0.08$)$ are shown in S1 in the ESI. $\dagger$ The compositional analysis of the nanoprecipitate of $\mathrm{Sn}_{0.92} \mathrm{La}_{0.08}$ Te is shown in $\mathrm{S} 2$ in the ESI. $\dagger$

A greater understanding of the morphology of the samples on an atomic scale can be explained by TEM or HRTEM images as shown in Fig. 4(a-c). Pristine SnTe and La-doped SnTe show interplanar spacing, $d \sim 0.223 \mathrm{~nm}$ which corresponds to the (2 2 0) plane of rock-salt structure type SnTe. ${ }^{21}$ The composition $\mathrm{Sn}_{0.92} \mathrm{La}_{0.08} \mathrm{Te}$ shows the presence of grain boundaries, nanoprecipitates of La due to the solubility limit and defects although Fig. 4(c) represents that all the three compositions of La-doped SnTe exhibit defects of various length scales and grain boundaries. These grain boundaries along with the defects present in the sample contribute to phonon scattering and thus lattice thermal conductivity is reduced. Thus, the presence of point defects due to the mass defect of Sn and La, La nanoprecipitates, and mesoscale kink bands as discussed earlier scatters the entire spectrum of phonons, thereby reducing the lattice thermal conductivity.

\subsection{Temperature dependent thermoelectric properties}

The temperature-dependent thermoelectric properties of La-doped SnTe samples are shown in Fig. 5. The temperature dependence of $\sigma$ of $\mathrm{Sn}_{1-x} \mathrm{La}_{x} \mathrm{Te}(x=0,0.04,0.06$, and 0.08$)$ is shown in Fig. 5(a)

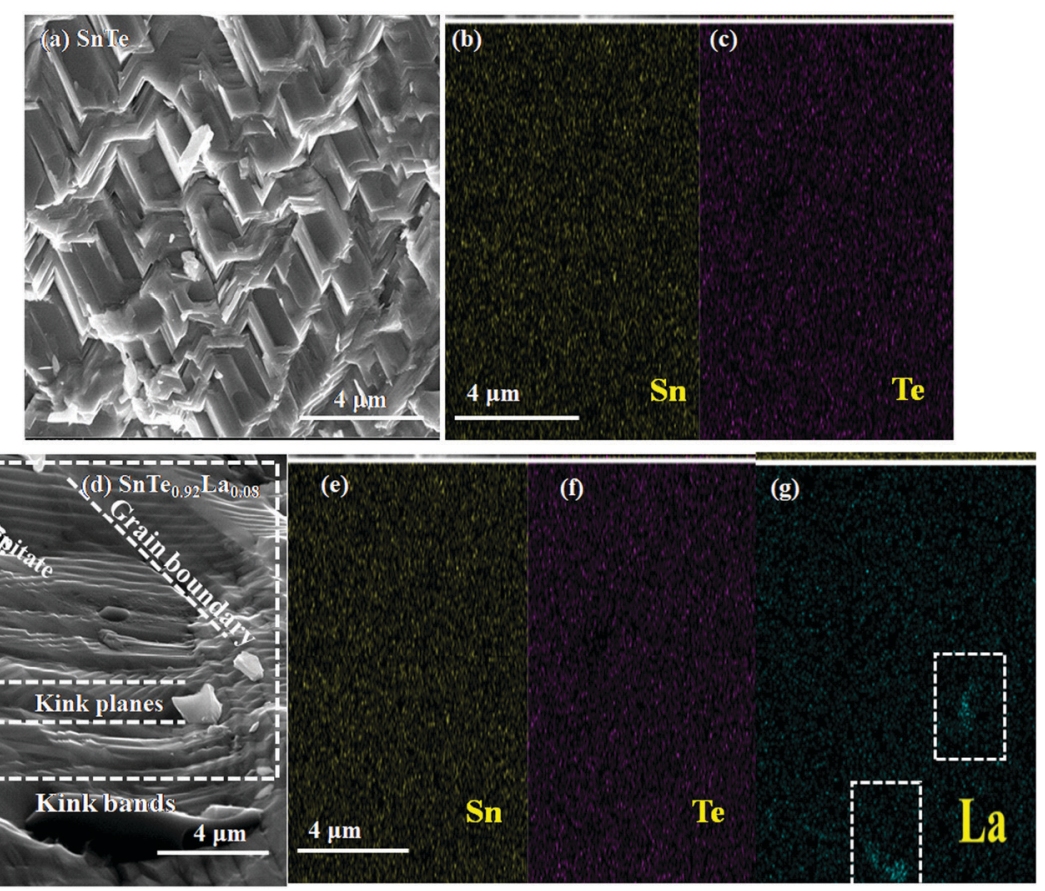

Fig. 3 SEM with EDX mapping images of $(a-c) S n T e,(d-g) S n_{0.92} L_{0} a_{0.08} T e$ samples after SPS. 

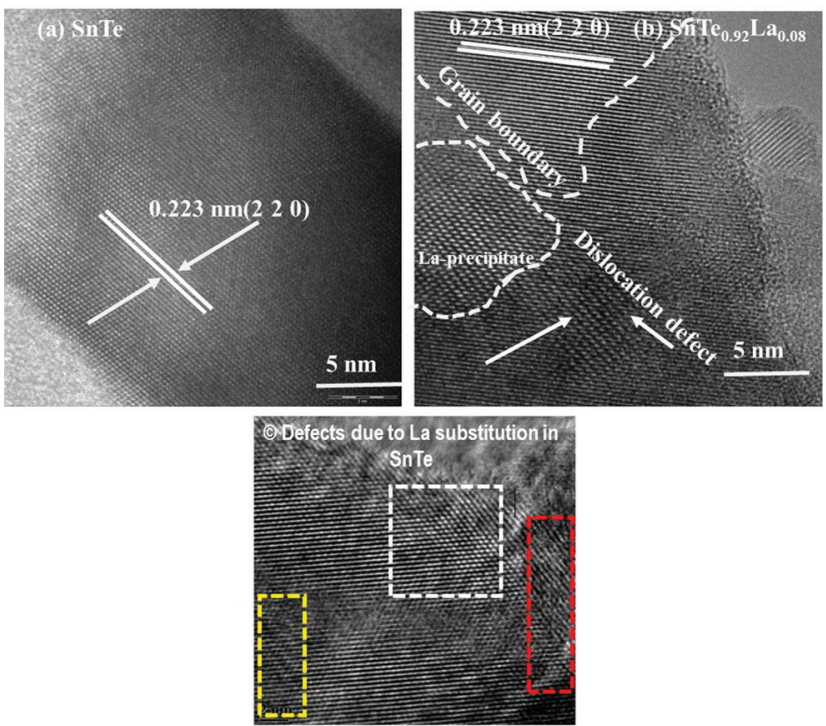

Fig. 4 TEM images of (a) SnTe, (b) $\mathrm{Sn}_{0.92} \mathrm{La}_{0.08} \mathrm{Te}$ and (c) different types of structural defects present in $\mathrm{Sn}_{1-\chi} \mathrm{La}_{x} \mathrm{Te}$ samples after SPS.

and all the samples show the behaviour of degenerate semiconductors where the $\sigma$ decreases with increase in the temperature. As expected pristine SnTe sample, demonstrates a very high electrical conductivity of $\sim 7740 \mathrm{mho} \mathrm{cm}^{-1}$ at room temperature due to $\mathrm{Sn}$ vacancy concentration. Based on the observation of the $\sigma v s$. T plot, where the conductivity value decreases, especially at room tem-

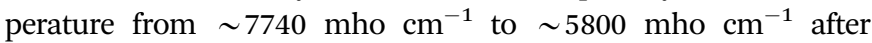
electron counter-doping, we conclude that La atoms should be a p-type dopant similar to $\mathrm{In}, \mathrm{Ga}, \mathrm{Bi}$ and $\mathrm{Sb}$ as electron dopants (counter-doping) which reduces the hole concentration. ${ }^{37}$ The intrinsic SnTe is p-type due to the $\mathrm{Sn}$ vacancies. These vacancies create empty electronic states and thus behave like a p-type dopant. When SnTe was doped with La, the La atoms first fill the "Sn" vacancies. Thus, despite being a p-type dopant, they are not as "robust" as Sn vacancies, in the sense that they induce fewer holes. Thus, at a low doping level, the p-type charge concentration i.e. holes decreases. However, as the doping level increases, at some point, all the Sn vacancies will be filled by La and beyond that point, excess La atoms substitute for Sn and the p-type charge carrier will again start increasing. However, when the solubility limit of La in SnTe will be achieved, the excess La atom will get precipitated and acts as a donor which will thus subsequently decrease the hole carrier concentration. The fact that the electrical conductivity decreases all the way indicates that the La atoms affected the hole mobility significantly and can be attributed to increased effective mass and scattering due to impurities. ${ }^{32}$

The temperature-dependent $S$ for all samples is shown in Fig. 5(b). All the samples exhibit a positive Seebeck coefficient value which indicates p-type conduction in the temperature range of 300 to $823 \mathrm{~K}$. No bipolar conduction was apparent even up to $823 \mathrm{~K}$, in all the compositions despite having a small band-gap of $0.18 \mathrm{eV}$ for SnTe. For all the samples, the Seebeck coefficient maxima shift to higher temperatures with rising La-fraction, but no Seebeck coefficient saturation (i.e. onset of bipolar diffusion) was observed. This observation is consistent with the relationships of band gap $\left(E_{\mathrm{g}}\right)$ and the temperature $T_{\max }$ at which $S_{\max }$ occurs (eqn (3)): ${ }^{10}$

$$
E_{\mathrm{g}}=2 e S_{\max } T_{\max }
$$

where $e$ is the electron charge and the plot is shown in S3 in the ESI. $\dagger$ The downturn in the Seebeck coefficient at high temperature is widely observed in $\mathrm{V}_{2}-\mathrm{VI}_{3}$ and IV-VI $(\mathrm{V}=\mathrm{Bi}$, $\mathrm{Sb}$; IV = Ge, Sn, Pb; VI = S, Se, Te) narrow gap thermoelectric systems. These bipolar effects degrade the Seebeck coefficients because the thermally excited minority carriers have Seebeck coefficients of the opposite sign and offset those of the majority ones. Therefore, bipolar effects limit the $\mathrm{z} T$ at higher temperatures. However, the bipolar effect in SnTe-based thermoelectric

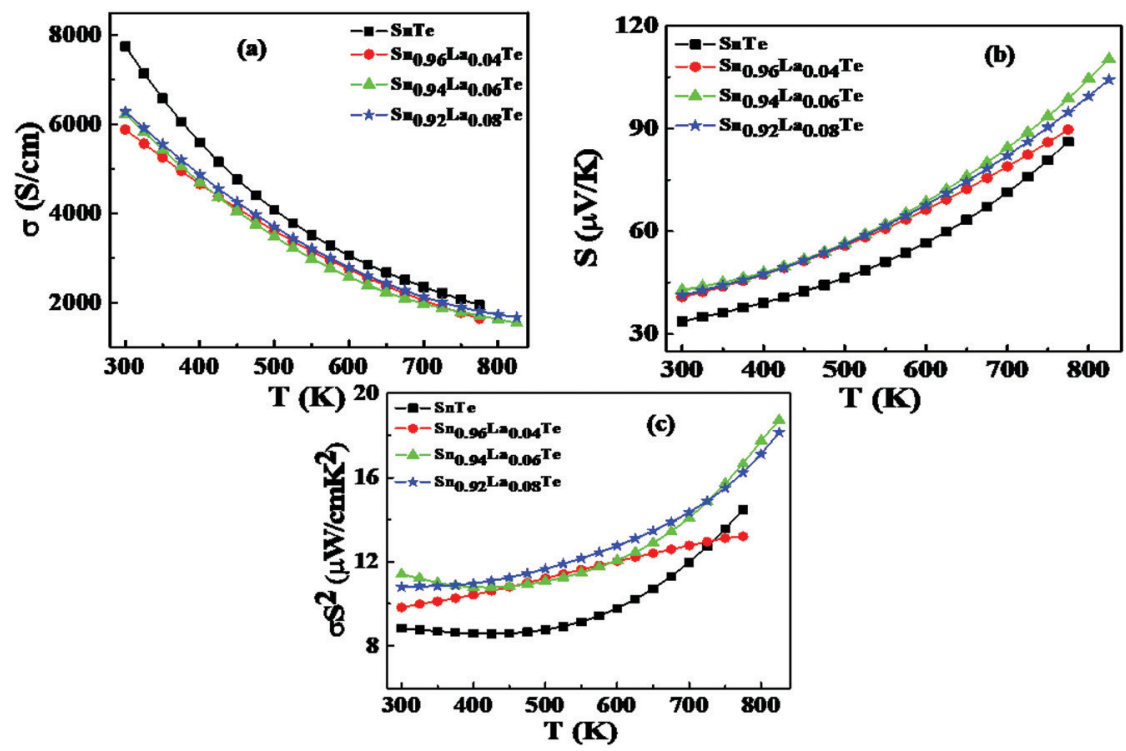

Fig. 5 Temperature dependence of (a) electrical conductivity, (b) Seebeck coefficient and (c) power factor for $\operatorname{Sn}_{1-x} \operatorname{La}_{x} T e(x=0,0.04,0.06$, and 0.08$)$. 
materials as reflected in the Seebeck coefficient (Fig. 5(b)) is not pronounced as in PbQ and $\mathrm{V}_{2}-\mathrm{VI}_{3}$ systems because of the very high hole concentration (majority carriers) of SnTe, which is similar to GeTe. ${ }^{38}$ The room temperature $S$ for pristine SnTe is $\sim 32 \mu \mathrm{V} \mathrm{K} \mathrm{K}^{-1}$ which increased to $\sim 45 \mu \mathrm{V} \mathrm{K} \mathrm{K}^{-1}$ for La-doped samples although the value of the Seebeck coefficient changes negligibly with the concentration of La atoms. The enhancement is $40 \%$ over pristine SnTe. This is attributed to the fact that analogous to the In doped SnTe which introduces resonant levels, La-doped SnTe introduces a Fermi level near the edge of the valence band. This is possible due to the interaction of the energetical proximity of the $6 \mathrm{~s}$ orbital of the La atom and the $5 \mathrm{p}$ orbital of Te atom. These two orbitals hybridize to form an s-p antibonding state. As a result, the electronic density of states near the top of the valence band is significantly increased, thereby enhancing the Seebeck coefficient at room temperature. Furthermore, we have shown the room temperature Pisarenko plot $S$ (shown in Fig. 6) of La-doped SnTe (our work) as a function of carrier concentration to clarify the origin of the enhanced Seebeck coefficient. The solid curve was calculated on the basis of two parabolic band models as described by Zhang et $a l .{ }^{32}$ The valence band model (VBM) takes into account the non-parabolicity of the light hole band (L-band) and provides a best quantitative fit to all the Seebeck coefficient values, expect for the In-doped or La-doped samples whose deviation implies an alternative mechanism through which the band structure of pure SnTe near the band edge is altered and one possible mechanism is the introduction of resonance levels.

However, at high temperatures, the increase in the Seebeck coefficient is not very prominent between the pristine SnTe snd the La-doped SnTe. This is because resonance levels are known for their ability to enhance the density-of-states and thus the Seebeck coefficient only near room temperature. Furthermore,

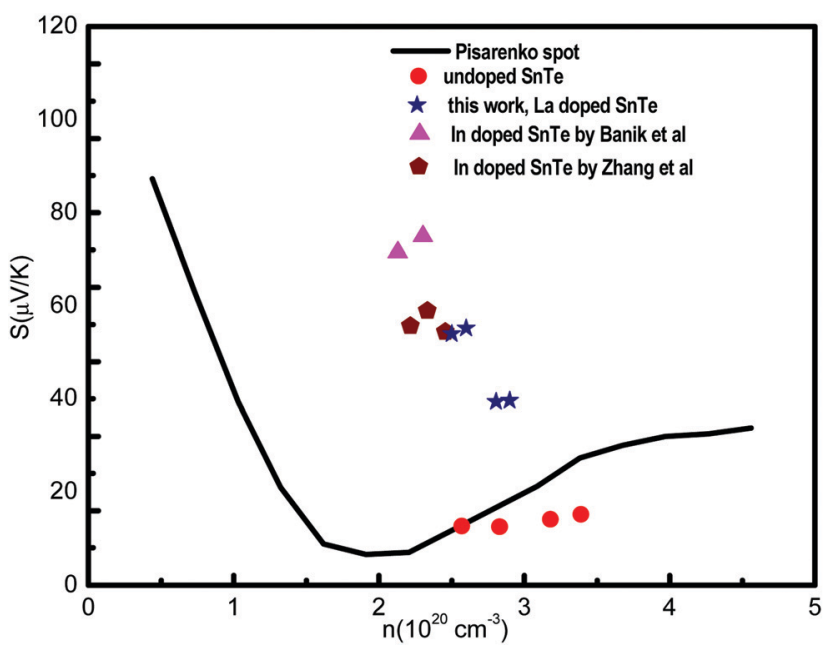

Fig. 6 Room temperature Seebeck coefficient as a function of carrier concentration of the present $\mathrm{Sn}$-based samples. The solid line is a Pisarenko plot at $300 \mathrm{~K}$ computed using the two-valence band model. The experimental data on undoped SnTe, ${ }^{32}$ In-doped SnTe by Zhang et al. ${ }^{32}$ and Banik et al. ${ }^{39}$ are in the inset for comparison. as discussed earlier, at a higher temperatures, the Fermi energy level is lowered and pushed deeper into the valence band of SnTe which in turn mitigates the phenomenon of the resonance effect, meanwhile facilitating the participation of the heavy hole band, although partially, in the carrier transport. ${ }^{38}$ Here, the enhancement in the Seebeck effect at high temperatures, essentially the heavy hole, $\Sigma$ band contributes at high temperature once the Sn-vacancies are removed substantially. This phenomenon opens the band gap and diminishes the band offset between the light valence band (L-band) and the heavy valence band ( $\Sigma$-band). The band-gap enlargement reduces the minority charge carriers at elevated temperature and suppresses the bipolar diffusion, i.e. the material is eligible for thermoelectric applications for a wider range of temperature. ${ }^{38}$ Since, at high temperature, the increase in the Seebeck coefficient is not very high for La-doped SnTe samples as compared to $\mathrm{Hg} / \mathrm{Cd} /$ $\mathrm{Mn} / \mathrm{Mg} / \mathrm{Ca}$ doped SnTe, it can be assumed that the La-doping in SnTe pushes the Pisarenko plot to region II, where the heavy valence band ( $\Sigma$-band) contributes partially alongwith the L-band in the transport properties. ${ }^{37}$ Thus, according to Kane-band theory, the conduction band edge and the valence band edges in SnTe are at the $L$-point, with a narrow band-gap of $0.18 \mathrm{eV}$. The small energy gap causes a highly non-parabolic light hole band with a small band edge density of states (DOS) effective mass of $m^{*} \sim$ $0.168 m_{\mathrm{e}}$. This narrow energy gap is also a probable reason for the introduction of resonance state upon doping. Moreover, a flatter, heavy-mass region of the valence band lies below the valence band edge, with the energy difference between the lighthole and heavy-hole maxima estimated to be in the range of $\Delta E \sim 0.3-0.4 \mathrm{eV}$ with an effective mass of $\sim 1.92 \mathrm{~m}_{\mathrm{e}} \cdot{ }^{18}$ Fig. 5(c) shows the temperature dependence of the power factor for $\mathrm{Sn}_{1-x} \mathrm{La}_{x} \mathrm{Te}(x=0,0.04,0.06$ and 0.08$)$ samples. It can be seen that the La-doped samples exhibit a higher power factor over a very broad temperature range because of the largely improved Seebeck coefficient. The power factor enhancement to $11.5 \mu \mathrm{W}$ $\mathrm{cm}^{-1} \mathrm{~K}^{-2}$ at $300 \mathrm{~K}$ for La-doped samples $(x \geq 0.06)$ is highest and the increase is $35 \%$ in comparison to the pristine SnTe. The highest power factor of $19 \mu \mathrm{W} \mathrm{cm}{ }^{-1} \mathrm{~K}^{-2}$ was achieved at $823 \mathrm{~K}$ for $\mathrm{Sn}_{0.94} \mathrm{La}_{0.06} \mathrm{Te}$ which is comparable with the other doped SnTe samples having high $z T$ values. ${ }^{39}$ Moreover, this is the highest power factor value achieved compared to other reported values for lanthanides. ${ }^{40}$

\subsection{Thermal transport properties}

The total thermal conductivity, $\kappa_{\text {total }}$ and the calculated lattice thermal conductivity, $\kappa_{\mathrm{L}}$ of $\mathrm{Sn}_{1-x} \mathrm{La}_{x} \mathrm{Te}(x=0,0.04,0.06$, and 0.08) samples are shown in Fig. 7(a and b). The overall thermal conductivity at room temperature is decreased from $\sim 7.5 \mathrm{~W} \mathrm{~m}^{-1} \mathrm{~K}^{-1}$ for pristine SnTe to $\sim 5.5 \mathrm{~W} \mathrm{~m}^{-1} \mathrm{~K}^{-1}$ for $\mathrm{Sn}_{0.96} \mathrm{La}_{0.04} \mathrm{Te}$. The thermal conductivities of all the La-doped samples are lower than that of the undoped SnTe which comes from the reduction of the electronic part of the thermal conductivity (due to the decrease in the hole concentration. ${ }^{30}$ The electronic contribution of the thermal conductivity is given by Wiedemann-Franz law, Where $T$ is the absolute temperature, $\sigma$ is the electrical conductivity, and $L$ is the Lorenz number. 

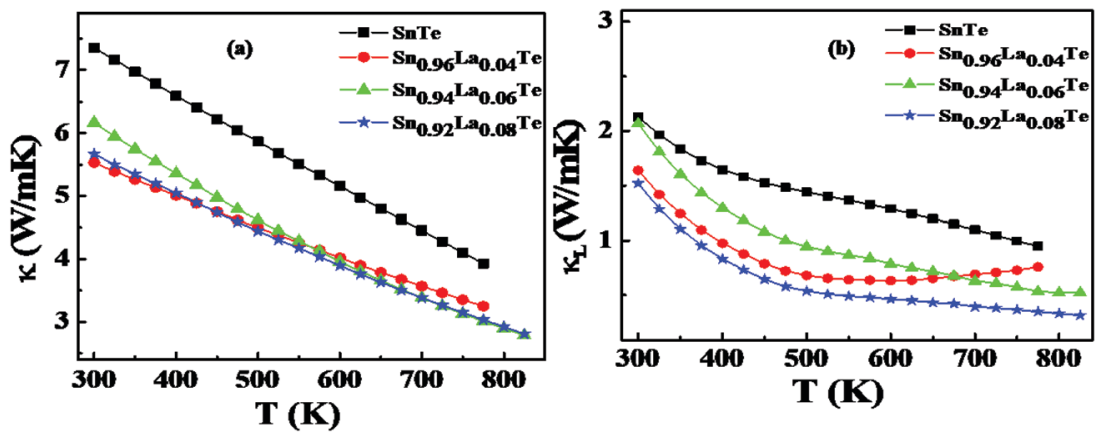

Fig. 7 Temperature-dependent (a) and (b) of $\mathrm{Sn}_{1-x} \mathrm{La}_{x} \mathrm{Te}(x=0,0.04,0.06$, and 0.08$)$ samples.

For the two-band model, $L$ is estimated as ((eqn (4) and (5))

$$
L=\frac{L_{\mathrm{L}} \sigma_{\mathrm{L}} T+L_{\Sigma} \sigma_{\Sigma} T+\kappa_{\text {bipolar }}}{\sigma_{\mathrm{L}} T+\sigma_{\Sigma} T}
$$

where,

$$
\kappa_{\text {bipolar, } \mathrm{L}-\Sigma}=\frac{T\left(\sigma_{\mathrm{L}} \alpha_{\mathrm{L}}^{2}+\sigma_{\Sigma} \alpha_{\Sigma}^{2}-\left(\sigma_{\mathrm{L}} \alpha_{\mathrm{L}}+\sigma_{\Sigma} \alpha_{\Sigma}\right)^{2}\right)}{\sigma_{\mathrm{L}}+\sigma_{\Sigma}}
$$

The above equation includes only bipolar effects within the two valence bands-electron-hole bipolar effects (most commonly thought of) are not considered in this Lorenz number calculation. ${ }^{30}$ However, due to the non-parabolicity and complexity of the valence band structure around the Fermi level for p-type SnTe, the exact determination of $L$ is difficult. An estimation of $L$ can be made using a single parabolic band (SPB) model considering acoustic phonon scattering, resulting in $L$ with a deviation of less than $10 \%$ as compared with a more rigorous single nonparabolic band and multiple band model calculations. ${ }^{30}$ However, if we carefully observe the reduction in the total thermal conductivity due to La-doping, the reduction effect is weakened at high temperature. The $\kappa_{\text {lat }}$ conductivity at high temperature is determined mainly by a large number of low wavelength phonons. It is well-known that low wavelength phonons are mostly scattered by the atomic scale point defects due to mass and strain field fluctuations. For the alloys with point defects, the scattering factor $(A)$ could be expressed by the following eqn (6) when the measuring temperature is beyond $650 \mathrm{~K}^{41}$

$$
A=\frac{\Omega_{0}}{4 \Pi \nu^{2}} x(1-x)\left(\frac{\Delta M}{M}\right)^{2}
$$

where $\Omega_{0}, \nu, x, \Delta M, M$ are the volume of the unit cell, the lattice sound velocity, the fraction of guest atom, the atomic mass difference between the guest and the host, and the average mass of the cell, respectively. The higher the atomic mass difference between the doping atoms and the host atoms $(\Delta M)$ is, the larger the value of $\Delta M / M$ would be and thus the smaller is the lattice thermal conductivity. Thus, all the La-doped samples exhibit low lattice thermal conductivity in comparison to the pristine SnTe, due to the reasonably high atomic mass unit (a.m.u.) difference of La (a.m.u. of $\mathrm{La}=138.905$ ) and $\mathrm{Sn}$ (a.m.u. of $\mathrm{Sn}=118.71$ ). Moreover, it is also seen that $\mathrm{La}(x=0.08)$ shows the least $\kappa_{\text {lat }}$ value.
Finally, it is confirmed that the $\kappa_{\text {total }}$ of La-doped SnTe samples are significantly suppressed in comparison to the pristine SnTe. The reduction of $\kappa_{\text {total }}$ is mainly attributed to the mass fluctuation caused by point defects and grain boundaries scattering as reported for other thermoelectric materials and $^{7,22,29}$ the same is evident from Fig. 7(b).

The thermoelectric $z T$ is calculated using the measured $\sigma, S$ and $\kappa_{\text {total }}$. Fig. 8 shows the temperature-dependent of thermoelectric $z T$ of $\mathrm{Sn}_{1-x} \mathrm{La}_{x} \mathrm{Te}(x=0,0.04,0.06$ and 0.08$)$. The samples showed a systematic increase in $z T$ with increasing La content in SnTe at room temperature. The $z T$ value of $\sim 0.6$ at $823 \mathrm{~K}$ has been obtained for $\mathrm{Sn}_{0.94} \mathrm{La}_{0.06} \mathrm{Te}$, which is $\sim 96 \%$ higher than the value 0.28 at $775 \mathrm{~K}$ of pristine SnTe and this value is higher than the reported value of other lanthanides $\left(\mathrm{Sn}_{0.94} \mathrm{Gd}_{0.06} \mathrm{Te}\right.$ and $\left.\mathrm{Sn}_{49.25} \mathrm{Te}_{49.25} \mathrm{Eu}_{1.5}\right){ }^{40,42}$

Finally, the TE device $z T$ was calculated using the equation, given a finite, temperature difference $\Delta T=T_{\mathrm{h}}-T_{\mathrm{c}}$, is then defined from the maximum efficiency $\eta$ of a single thermoelectric leg, is given by eqn (7):

$$
z T=\left(\frac{T_{\mathrm{h}}-T_{\mathrm{c}}(1-\eta)}{T_{\mathrm{h}}(1-\eta)-T_{\mathrm{c}}}\right)^{2}-1
$$

where the maximum conversion efficiency $(\eta)$ were estimated by using the eqn (8):

$$
\eta_{\max }=\frac{\Delta T}{T_{\mathrm{h}}}\left(\frac{\sqrt{z \bar{T}+1}-1}{\sqrt{z \bar{T}+1}+\frac{T_{\mathrm{c}}}{T_{\mathrm{h}}}}\right)
$$

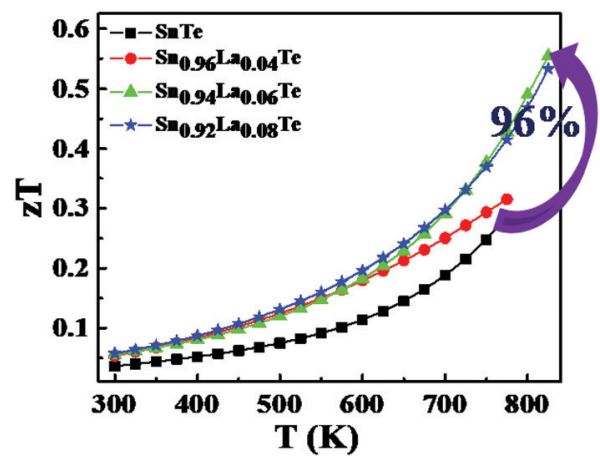

Fig. 8 Temperature dependent $z T$ of $S n_{1-x} \operatorname{La}_{x} T e(x=0$ to 0.08$)$ 

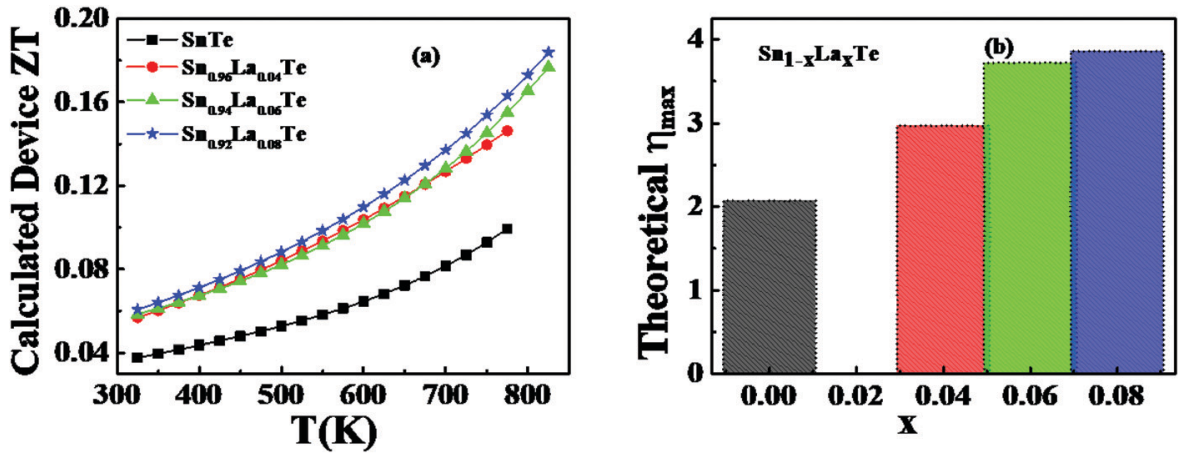

Fig. 9 (a) Temperature-dependent calculated device $z T$ (b) Theoretical maximum conversion efficiency in percentage.

from the temperature-dependent properties $S(T), \sigma(T)$, and $\kappa(T)$ between $T_{\mathrm{h}}$ and $T_{\mathrm{c}}$ as suggested by Snyder $e t a{ }^{43}{ }^{43}$ Furthermore, Fig. 9(a) shows the temperature-dependence of calculated device $z T$ of $\mathrm{Sn}_{1-x} \mathrm{La}_{x} \mathrm{Te}(x=0$ to 0.08$)$ and all the samples have shown a systematic improvement of device $z T$ with increasing La content in SnTe. Finally, the device $z T$ value of 0.17 at $823 \mathrm{~K}$ has been obtained for $\mathrm{Sn}_{0.92} \mathrm{La}_{0.08} \mathrm{Te}$, which is $\sim 88 \%$ higher than the value 0.09 at $775 \mathrm{~K}$ of pristine SnTe. The exact calculation of maximum efficiency is explained in the spreadsheet S.4.1 to S.4.4 for download in the ESI. $\dagger$ Also, Fig. 9(b) represents the theoretical maximum conversion efficiency of La-doped SnTe samples. Moreover, all the samples have shown a systematic increase in conversion efficiency with increasing La content in SnTe. Finally, the maximum conversion efficiency has been observed to be increased from 2.0 to $3.7 \%$ for $\mathrm{Sn}_{0.92} \mathrm{La}_{0.08} \mathrm{Te}$. The significant improvement in device efficiency is encouraging for considering these materials as a suitable alternative to $\mathrm{Pb}$-based chalcogenides.

\section{Conclusions}

In summary, La-doped SnTe samples are successfully synthesized by vacuum melting. The synthesized samples have shown a maximum solubility of 6 at\% La in SnTe; beyond that La, gets precipitated which was confirmed by microstructural investigations by SEM and TEM. Furthermore, the enhancement of the Seebeck coefficient and power factor for La-doped SnTe was proposed to be due to the creation of resonance states where the Fermi level lies in the valence band at room temperature. However, at high temperature the participation of the heavy valence band in the transport properties, and the presence of defects of all length scale which significantly enhances the phonon scattering resulted in the $z T$ value of $\sim 0.6$ at $823 \mathrm{~K}$ for $\mathrm{Sn}_{0.94} \mathrm{La}_{0.06} \mathrm{Te}$, which is $\sim 96 \%$ higher for pristine SnTe. Also, the device $z T$ value of 0.17 at $823 \mathrm{~K}$ was obtained for $\mathrm{Sn}_{0.92} \mathrm{La}_{0.08} \mathrm{Te}$, which is $\sim 88 \%$ higher than the value 0.09 at $773 \mathrm{~K}$ of pristine SnTe. The current investigation resembles that there is a large scope to enhance thermoelectric performance for SnTe material by using the heavier lanthanide elements.

\section{Conflicts of interest}

There is no conflict of interest.

\section{Acknowledgements}

FRGS no. GGSIPU/DRC/FRGS/2019/1553/11. The authors sincerely acknowledge Director CSIR-NPL, India, and BRNS (Board of Research in Nuclear Sciences), Department of Atomic Energy for their experimental and financial support (Sanction No. 37(3)/14/ 22/2016-BRNS India), respectively. The authors from SSSIHL thank the founder Chancellor Bhagawan Sri Sathya Sai Baba and Trustees/Administrators for providing characterization facilities at Central Research Instruments Facility (SSSIHL-CRIF).

\section{References}

1 Z. Hou, Y. Xiao and L.-D. Zhao, Investigation on carrier mobility when comparing nanostructures and bands manipulation, Nanoscale, 2020, 12, 12741-12747.

2 W. Wunderlich, H. Ohta and K. Koumoto, Enhanced effective mass in doped $\mathrm{SrTiO}_{3}$ and related perovskites, Phys. B, 2009, 404, 2202-2212.

3 D. M. Rowe, CRC handbook of thermoelectrics, CRC Press, 2018.

4 Z. M. Gibbs, F. Ricci, G. Li, H. Zhu, K. Persson and G. Ceder, et al., Effective mass and Fermi surface complexity factor from ab initio band structure calculations, npj Comput. Mater., 2017, 3, 8.

5 S. Kasap, C. Koughia and H. E. Ruda, Electrical conduction in metals and semiconductors, Springer Handbook of Electronic and Photonic Materials, Springer, 2017, p. 1.

$6 \mathrm{~K}$. Hess, Advanced theory of semiconductor devices, 2000.

7 S. Bhattacharya, A. Bohra, R. Basu, R. Bhatt, S. Ahmad, K. N. Meshram, A. K. Debnath, S. K. Sarkar, M. Navaneethan, Y. Hayakawa, D. K. Aswal and S. K. Gupta, High thermoelectric performance of $\left(\mathrm{AgCrSe}_{2}\right)_{0.5}\left(\mathrm{CuCrSe}_{2}\right)_{0.5}$ nano-composites having all-scale natural hierarchical architectures., J. Mater. Chem. A, 2014, 2, 17122-17129.

8 R. Basu, S. Bhattacharya, R. Bhatt, M. Roy, S. Ahmad, A. Singh, M. Navaneethan, Y. Hayakawa, D. K. Aswal and S. K. Gupta, Improved thermoelectric performance of hot pressed nanostructured n-type SiGe bulk alloys, J. Mater. Chem. A, 2014, 2, 6922-6930.

9 K. Biswas, J. He, I. D. Blum, C.-I. Wu, T. P. Hogan, D. N. Seidman, V. P. Dravid and M. G. Kanatzidis, High-performance 
bulk thermoelectrics with all-scale hierarchical architectures, Nature, 2012, 489, 414-418.

10 L.-D. Zhao, H. Wu, S. Hao, C.-I. Wu, X. Zhou, K. Biswas, J. He, T. P. Hogan, C. Uher, C. Wolverton, V. P. Dravid and M. G. Kanatzidis, All-scale hierarchical thermoelectrics: MgTe in PbTe facilitates valence band convergence and suppresses bipolar thermal transport for high performance, Energy Environ. Sci., 2013, 6, 3346-3355.

11 G. Tan, F. Shi, S. Hao, H. Chi, T. P. Bailey, L.-D. Zhao, C. Uher, C. Wolverton, V. P. Dravid and M. G. Kanatzidis, Valence Band Modification and High Thermoelectric Performance in SnTe Heavily Alloyed with MnTe, J. Am. Chem. Soc., 2015, 137, 11507-11516.

12 Z. Ma, C. Wang, Y. Chen, L. Li, S. Li, J. Wang and H. Zhao, Ultra-high thermoelectric performance in SnTe by the integration of several optimization strategies, Mater. Today Phys., 2021, 17, 100350.

13 R. Moshwan, W.-D. Liu, X.-L. Shi, Y.-P. Wang, J. Zou and Z.-G. Chen, Realizing high thermoelectric properties of SnTe via synergistic band engineering and structure engineering, Nano Energy, 2019, 65, 104056.

14 D. K. Bhat and U. S. Shenoy, Zn: a versatile resonant dopant for SnTe thermoelectrics, Mater. Today Phys., 2019, 11, 100158.

15 R. D. Schmidt, E. D. Case, J. E. Ni, R. M. Trejo, E. LaraCurzio, R. J. Korkosz and M. G. Kanatzidis, High-temperature elastic moduli of thermoelectric $\mathrm{SnTe}_{1 \pm x-y} \mathrm{SiC}$ nanoparticulate composites, J. Mater. Sci., 2013, 48, 8244-8258.

16 A. Banik, U. S. Shenoy, S. Anand, U. V. Waghmare and K. Biswas, Mg Alloying in SnTe Facilitates Valence Band Convergence and Optimizes Thermoelectric Properties, Chem. Mater., 2015, 27, 581-587.

17 X. J. Tan, H. Z. Shao, J. He, G. Q. Liu, J. T. Xu, J. Jiang and H. C. Jiang, Band engineering and improved thermoelectric performance in $\mathrm{M}$-doped SnTe ( $\mathrm{M}=\mathrm{Mg}, \mathrm{Mn}, \mathrm{Cd}$, and $\mathrm{Hg})$, Phys. Chem. Chem. Phys., 2016, 18, 7141-7147.

18 G. Tan, W. G. Zeier, F. Shi, P. Wang, G. J. Snyder, V. P. Dravid and M. G. Kanatzidis, High Thermoelectric Perfor-

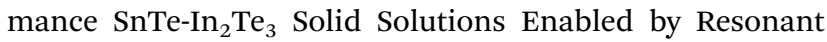
Levels and Strong Vacancy Phonon Scattering, Chem. Mater., 2015, 27, 7801-7811.

19 G. Tan, L.-D. Zhao, F. Shi, J. W. Doak, S.-H. Lo, H. Sun, C. Wolverton, V. P. Dravid, C. Uher and M. G. Kanatzidis, High Thermoelectric Performance of p-Type SnTe via a Synergistic Band Engineering and Nanostructuring Approach, J. Am. Chem. Soc., 2014, 136, 7006-7017.

20 S. Ahmad, A. Singh, S. Bhattacharya, M. Navaneethan, R. Basu, R. Bhatt, P. Sarkar, K. N. Meshram, A. K. Debnath, K. P. Muthe and D. K. Aswal, Band Convergence and Phonon Scattering Mediated Improved Thermoelectric Performance of SnTe-PbTe Nanocomposites, ACS Appl. Energy Mater., 2020, 3, 8882-8891.

21 S. Ahmad, A. Singh, S. Bhattacharya, M. Navaneethan, R. Basu, R. Bhatt, P. Sarkar, K. N. Meshram, K. P. Muthe and S. Vitta, Remarkable Improvement of Thermoelectric Figure-of-Merit in SnTe through In Situ-Created Te Nanoinclusions, ACS Appl. Energy Mater., 2020, 3, 7113-7120.
22 D. Sarkar, T. Ghosh, A. Banik, S. Roychowdhury, D. Sanyal and K. Biswas, Highly Converged Valence Bands and Ultralow Lattice Thermal Conductivity for High-Performance SnTe Thermoelectrics, Angew. Chem., Int. Ed., 2020, 59, 11115-11122.

23 J.-W. Zhang, Z.-W. Wu, B. Xiang, N.-N. Zhou, J.-L. Shi and J.-X. Zhang, Ultralow Lattice Thermal Conductivity in SnTe by Incorporating InSb, ACS Appl. Mater. Interfaces, 2020, 12, 21863-21870.

24 Y. Pei, X. Shi, A. LaLonde, H. Wang, L. Chen and G. J. Snyder, Convergence of electronic bands for highperformance bulk thermoelectrics, Nature, 2011, 473, 66-69.

25 F. Guo, B. Cui, H. Geng, Y. Zhang, H. Wu, Q. Zhang, B. Yu, S. J. Pennycook, W. Cai and J. Sui, Simultaneous Boost of Power Factor and Figure-of-Merit in In-Cu Codoped SnTe, Small, 2019, 15, 1902493.

26 A. Doi, S. Shimano, D. Inoue, T. Kikitsu, T. Hirai, D. Hashizume, Y. Tokura and Y. Taguchi, Band engineering, carrier density control, and enhanced thermoelectric performance in multidoped SnTe, APL Mater., 2019, 7, 091107.

27 X. Guo, Z. Chen, J. Tang, F. Zhang, Y. Zhong, H. Liu and R. Ang, Thermoelectric transport properties in Bi-doped SnTe-SnSe alloys, Appl. Phys. Lett., 2020, 116, 103901.

28 J. P. Heremans, B. Wiendlocha and A. M. Chamoire, Resonant levels in bulk thermoelectric semiconductors, Energy Environ. Sci., 2012, 5, 5510-5530.

29 M. Zhou, G. J. Snyder, L. Li and L.-D. Zhao, Lead-free tin chalcogenide thermoelectric materials, Inorg. Chem. Front., 2016, 3, 1449-1463.

30 M. Zhou, Z. M. Gibbs, H. Wang, Y. Han, C. Xin, L. Li and G. J. Snyder, Optimization of thermoelectric efficiency in SnTe: the case for the light band, Phys. Chem. Chem. Phys., 2014, 16, 20741-20748.

31 X. Chen, D. Parker and D. J. Singh, Importance of nonparabolic band effects in the thermoelectric properties of semiconductors, Sci. Rep., 2013, 3, 1-6.

32 Q. Zhang, B. Liao, Y. Lan, K. Lukas, W. Liu, K. Esfarjani, C. Opeil, D. Broido, G. Chen and Z. Ren, High thermoelectric performance by resonant dopant indium in nanostructured SnTe, Proc. Natl. Acad. Sci. U. S. A., 2013, 110, 13261-13266.

33 X. Tan, X. Tan, G. Liu, J. Xu, H. Shao, H. Hu, M. Jin, H. Jiang and J. Jiang, Optimizing the thermoelectric performance of In-Cd codoped SnTe by introducing Sn vacancies, J. Mater. Chem. C, 2017, 5, 7504-7509.

34 X. Tan, G. Liu, J. Xu, X. Tan, H. Shao, H. Hu, H. Jiang, Y. Lu and J. Jiang, Thermoelectric properties of In-Hg co-doping in SnTe: Energy band engineering, J. Materiomics, 2018, 4, 62-67.

35 Q. Yang, T. Lyu, Z. Li, H. Mi, Y. Dong, H. Zheng, Z. Sun, W. Feng and G. Xu, Realizing widespread resonance effects to enhance thermoelectric performance of SnTe, J. Alloys Compd., 2021, 852, 156989.

36 L. Yang, Q. Zhang, Z. Wei, Z. Cui, Y. Zhao, T. T. Xu, J. Yang and D. Li, Kink as a New Degree of Freedom to Tune the Thermal Conductivity of Si Nanoribbons, J. Appl. Phys., 2019, 126, 155103. 
37 L.-D. Zhao, X. Zhang, H. Wu, G. Tan, Y. Pei, Y. Xiao, C. Chang, D. Wu, H. Chi and L. Zheng, Enhanced thermoelectric properties in the counter-doped SnTe system with strained endotaxial SrTe, J. Am. Chem. Soc., 2016, 138, 2366-2373.

38 H. Wu, C. Chang, D. Feng, Y. Xiao, X. Zhang, Y. Pei, L. Zheng, D. Wu, S. Gong and Y. Chen, Synergistically optimized electrical and thermal transport properties of SnTe via alloying high-solubility MnTe, Energy Environ. Sci., 2015, 8, 3298-3312.

39 A. Banik, S. Roychowdhury and K. Biswas, The journey of tin chalcogenides towards high-performance thermoelectrics and topological materials, Chem. Commun., 2018, 54, 6573-6590.
40 X. Wang, K. Guo, I. Veremchuk, U. Burkhardt, X. Feng, J. Grin and J. Zhao, Thermoelectric properties of Eu- and Na-substituted SnTe, J. Rare Earths, 2015, 33, 1175-1181.

41 Z. Ma, C. Wang, J. Lei, D. Zhang, Y. Chen, J. Wang, Z. Cheng and Y. Wang, High Thermoelectric Performance of SnTe by the Synergistic Effect of Alloy Nanoparticles with Elemental Elements, ACS Appl. Energy Mater., 2019, 2, 7354-7363.

42 L. Zhang, J. Wang, Z. Cheng, Q. Sun, Z. Li and S. Dou, Leadfree SnTe-based thermoelectrics: enhancement of thermoelectric performance by doping with $\mathrm{Gd} / \mathrm{Ag}, \mathrm{J}$. Mater. Chem. A, 2016, 4, 7936-7942.

43 G. J. Snyder and A. H. Snyder, Figure of merit ZT of a thermoelectric device defined from materials properties, Energy Environ. Sci., 2017, 10, 2280-2283. 